\title{
Expression of insulin-like growth factor 2 in mesenchymal neoplasms
}

\author{
Sonja E Steigen ${ }^{1}$, David F Schaeffer ${ }^{2}$, Robert B West ${ }^{3}$ and Torsten O Nielsen ${ }^{2}$ \\ ${ }^{1}$ Department of Pathology, University Hospital of Northern Norway and University of Tromsø, Tromsø, \\ Norway; ${ }^{2}$ Department of Pathology and Genetic Pathology Evaluation Centre, University of British Columbia, \\ Vancouver, Canada and ${ }^{3}$ Department of Pathology, Stanford University Medical Center, Stanford, CA, USA
}

\begin{abstract}
The insulin-like growth factor (IGF) system plays an important role in the growth and development of cells and has been implicated in oncogenesis and tumor progression. Gene expression profiling studies on limited numbers of specimens have shown high expression of IGF2, encoding the activating ligand for this system, in gastrointestinal stromal tumors (GISTs) and in synovial sarcomas. This data may have concrete clinical implications, as several reports exist of patients with GISTs suffering from severe hypoglycemia, a predicted effect of IGF2. Furthermore, new drugs targeting IGF signaling are entering clinical trials. The purpose of this study is to survey IGF2 expression at the protein level on a broad number of mesenchymal tumors representing all major diagnostic classes. By immunostaining tissue microarrays, results were obtained for 51 diagnostic categories of bone and soft-tissue tumors representing 1288 cases. Distinct membranous and/or cytoplasmic IGF2 immunoreactivity was assessed according to published criteria. Solitary fibrous tumors had the highest expression. Of 20 tumor types represented by more than 10 cases, synovial sarcomas, myxoid liposarcomas, GISTs, malignant peripheral nerve sheath tumors, chondrosarcomas, undifferentiated pleomorphic sarcomas (MFH), Ewing's sarcomas and tenosynovial giant cell tumors showed high levels of expression in more than $20 \%$ of cases. Of the 445 GIST cases with clinical information, those with high expression of IGF2 had a significantly worse outcome than those with low or no expression. IGF2 protein expression among mesenchymal tumors is largely consistent with gene expression studies and suggests a potential for molecular therapy targeting the IGF signaling pathway system in these neoplasms.

Modern Pathology (2009) 22, 914-921; doi:10.1038/modpathol.2009.48; published online 1 May 2009
\end{abstract}

Keywords: gastrointestinal stromal tumors; GISTs; insulin-like growth factor; IGF2; tissue microarray; mesenchymal tumors

The insulin-like growth factor (IGF) signaling system plays a critical role in the growth and development of many tissues and regulates the overall growth, particularly in the prenatal period. ${ }^{1}$ Under normal physiological conditions, the balance between the expression and activities of the proteins involved in this pathway is tightly controlled. The IGF system has been implicated in various pathophysiological conditions, and is thought to play an especially prominent role in tumorigenesis. ${ }^{2-5}$ Throughout life, the majority of circulating ligands, IGF1 and IGF2, are produced by the liver, with the synthesis of IGF1 dependent on growth hormone but with the synthesis of IGF2 relatively independent of growth hormone. ${ }^{6}$ The IGFs circulate in the blood

Correspondence: Dr SE Steigen, Department of Pathology, University of Tromsø, University Hospital of Northern Norway, Sykehusveien, N-9038 Tromsø, Norway.

E-mail: sonja.steigen@unn.no

Received 20 November 2008; revised and accepted 17 December 2008; published online 1 May 2009 and are largely bound to one of the six IGF-binding proteins that modulate IGF activity. ${ }^{7}$ IGF1 and IGF2 both interact with the cell-surface IGF1 receptor (IGF1R), which possesses tyrosine kinase activity. ${ }^{8}$ IGF1R initiates signaling through two primary cascades, the MAP kinase and PI3 kinase pathways. IGF2 can also bind to IGF2R, ${ }^{9}$ which is regarded as a clearance receptor for IGF2 and decreases its effects. High levels of circulating IGFs and/or the downregulation of IGF2R have been shown to correlate with the development of cancer in the colon, breast and prostate. ${ }^{10-12}$ Some mesenchymal (non-islet cell) tumors produce and release an excessive amount of a prohormone form of IGF2 (often termed, 'big IGF2'). ${ }^{13}$

Clues about which tumor types are most likely to be dependent on IGF signaling can come from gene expression profiling studies. Publicly available datasets show consistently that IGF2 is very highly expressed in gastrointestinal stromal tumors (GISTs), indeed making it one of the strongest discriminators for this diagnosis. ${ }^{14-17}$ The supplemental 
data presented by Hernando et $a l^{17}$ show IGF2 to be the only tested signaling molecule to be relatively overexpressed in GIST to a greater extent than the KIT oncogene itself; indeed their data reveal IGF2 to be overexpressed by an impressive two to three orders of magnitude above most other signaling molecules.

The physiological actions of IGF2 include insulinlike effects ${ }^{18}$ and interestingly, several cases of patients with GIST suffering from severe hypoglycemia have been reported. ${ }^{19-23}$ Furthermore, a recent study by Braconi et $a^{24}$ indicates that IGF1 and IGF2 might help to predict disease outcome in GIST patients. The published expression profile data by Hernando et a ${ }^{17}$ also suggest that synovial sarcomas (and, to a lesser extent, fibrosarcomas and myxoid/ round cell liposarcomas) also express the IGF2 gene at very high levels, and high-grade sarcomas have been reported to express significantly more IGF2 than intermediate grade soft-tissue sarcomas. ${ }^{25}$

Gene expression findings need to be validated at the protein level, on larger numbers of specimens, with clinical correlation. Tissue microarrays are useful for such studies, permitting rapid testing of the diagnostic and prognostic utility of antibodies in large numbers of archival samples. ${ }^{26}$ In this study, we use tissue microarrays to evaluate the expression of IGF2 protein in a comprehensive collection of mesenchymal tumors. In the case of GISTs, a particularly large number of samples linked to clinical outcome were included, allowing assessment of IGF2 expression as a prognostic factor. These findings have a particular relevance in the context of new efforts to develop IGF pathway inhibitors $^{27}$ and to apply them to sarcomas (eg, SARC 011; http://www.sarctrials.com).

\section{Materials and methods}

\section{Patients, Samples and Tissue Microarrays}

The following tissue microarrays were used in this study:

1. Norwegian GIST and mesenchymal tumor array, 5 blocks/704 cases. ${ }^{28}$

2. Synovial sarcoma differential diagnosis array, 1 block/82 cases. $^{29}$

3. Chondroid tumor array, 1 block/121 cases. ${ }^{30}$

4. TA167 and TA170 soft-tissue tumor arrays, 2 blocks representing an expanded rebuild of earlier published multi-sarcoma arrays. ${ }^{31}$

5. MPNST differential diagnosis array, 1 block/172 cases. $^{32}$

6. Adipose tumor array, 1 block/58 cases, including 32 primary myxoid liposarcomas, 10 well-differentiated liposarcomas, 10 lipomas and 6 pleomorphic liposarcomas.

Each TMA comprises duplicate 0.6-mm cores generated from representative areas of formalinfixed, paraffin-embedded surgical excision blocks, which had been reviewed by at least two pathologists with appropriate subspecialty expertise.

\section{Immunohistochemical Staining and Scoring}

Four-micron sections from the arrays were stained with hematoxylin and eosin to confirm the presence of representative tumor in each core. Sections were stained with an anti-IGF2 rabbit polyclonal antibody from Abcam (Cambridge, MA, USA; cat\#ab9574) raised against a recombinant human IGF2 protein. This antibody recognizes both the prohormone form of IGF2 (also named 'big IGF2, $15 \mathrm{kDa}$ ') and the smaller IGF2 $(7.5 \mathrm{kDa})$. Immunostaining was performed on a Ventana Discovery XT (Tucson, AZ, USA) using a standard CC1 heat-induced epitope retrieval protocol (Tucson, AZ, USA), 1:100 dilution and DABMap detection system (Tucson, AZ, USA). A cervical carcinoma was used as a positive control with every staining run. To aid the analysis of the numerous tissue cores stained by immunohistochemistry, digital images were collected using a BLISS instrument (Bacus Laboratories, Lombard, IL, USA; http:// www.bacuslabs.com). Tissue cores with $>50$ interpretable tumor cells were scored on the basis of the percentage of positive tumor cells staining above background intensity in a membranous and/or cytoplasmic pattern according to published methodology. ${ }^{33}$ In tumor cells, the staining intensity was designated as either non-existent (0), weak (1), moderate (2) or strong (3). The number of cells was scored as either no cells stained (0), $<10 \%$ (1), $10-50 \%$ (2), $50-80 \%$ (3) or $>80 \%$ (4). The final score was calculated by multiplying these two variables. A score of $0-5$ was considered negative (IGF2 not overexpressed), and a score of 6 was considered positive (overexpression of IGF2) in accordance with Kobel et $a{ }^{33}$ Discrepant score results for duplicate cores, when present, were consolidated as the higher interpretable score. ${ }^{34}$

\section{Prognostic Evaluation}

To compare IGF2 staining in GISTs with established prognostic factors, whole section slides that were used for selecting representative TMA cores were used for a histological review of mitotic count in 50 consecutive high power fields. Clinicopathological data were present for all the GIST cases on the Norwegian GISTs and mesenchymal tumor array. ${ }^{28}$ The tumors were classified according to the consensus risk-group stratification system based on maximum tumor size, mitotic count (per 50 high powered fields) and location of tumor. ${ }^{35}$

\section{Statistical Analysis}

SPSS for Windows (Chicago, IL, USA; Version 14.0) was used for statistical analyses. For tumor types represented by $>10$ distinct patient samples, an 
overall immunostaining score was calculated as the mean of the scores among arrayed cases, with 95\% confidence interval. For correlations between IGF2 staining and size, mitosis and location of primary tumor, Pearson's $\chi^{2}$-test was performed. Survival curves were plotted using the Kaplan-Meier method, with significance assessed using log-rank tests. Cox proportional hazard regression was used for multivariate analysis with clinical and morphologic variables for 445 GISTs. For tumors without a known tumor size, this was categorized as a separate group for the purpose of retaining cases for analysis. Statistical significance was declared if the $P$-value was $<0.05$.

\section{Results}

\section{Immunostaining Observations}

IGF2 immunostaining was readily interpretable in the cytoplasm and on the membrane of positive mesenchymal cells, with low background staining (Figure 1). Primary image data for tumors included in this study are accessible at http://www.gpecimage.ubc.ca (login: igf2. password: abc123). Results on all tumor types represented by $>10$ cases are represented in Table 1a, and those with $\leq 10$ cases in Table $1 \mathrm{~b}$.

\section{GIST Tumors}

A total of 445 of the samples on the Norwegian abdominal mesenchymal tumor tissue microarray were KIT-positive and regarded as definite GISTs. Clinicopathological characteristics of these GISTs are listed in Table 2.

In 333 cases, the size of the tumors were known, and when combining this variable with number of mitoses and primary sites of tumor (the stomach, small bowel or other sites), a risk stratification using the method of Hornick and Fletcher ${ }^{35}$ could be estimated. A total of 70 tumors from the stomach were regarded as having a very low potential for malignancy, 36 had a low malignancy potential, 23 moderate and 48 a high malignancy potential. Of the tumors arising in the small bowel, only one was regarded as having a very low malignancy potential, 8 with low, 53 with moderate and 56 with a high malignancy potential.

There was an overall median survival time for patients with gastric GISTs of 5.5 years, compared with patients with tumors in the small bowel with a median of 3.2 years $(P<0.001)$. There was a correlation of IGF2 with risk stratification $(P=0.024)$, with twice as many of the low IGF2expressing tumors represented among the groups with very low and low malignancy potential. In this pre-imatinib cohort, the median overall survival of patients with tumors with low expression of IGF2 was 4.9 years, compared with patients with an overexpression of IGF2 who had a median survival
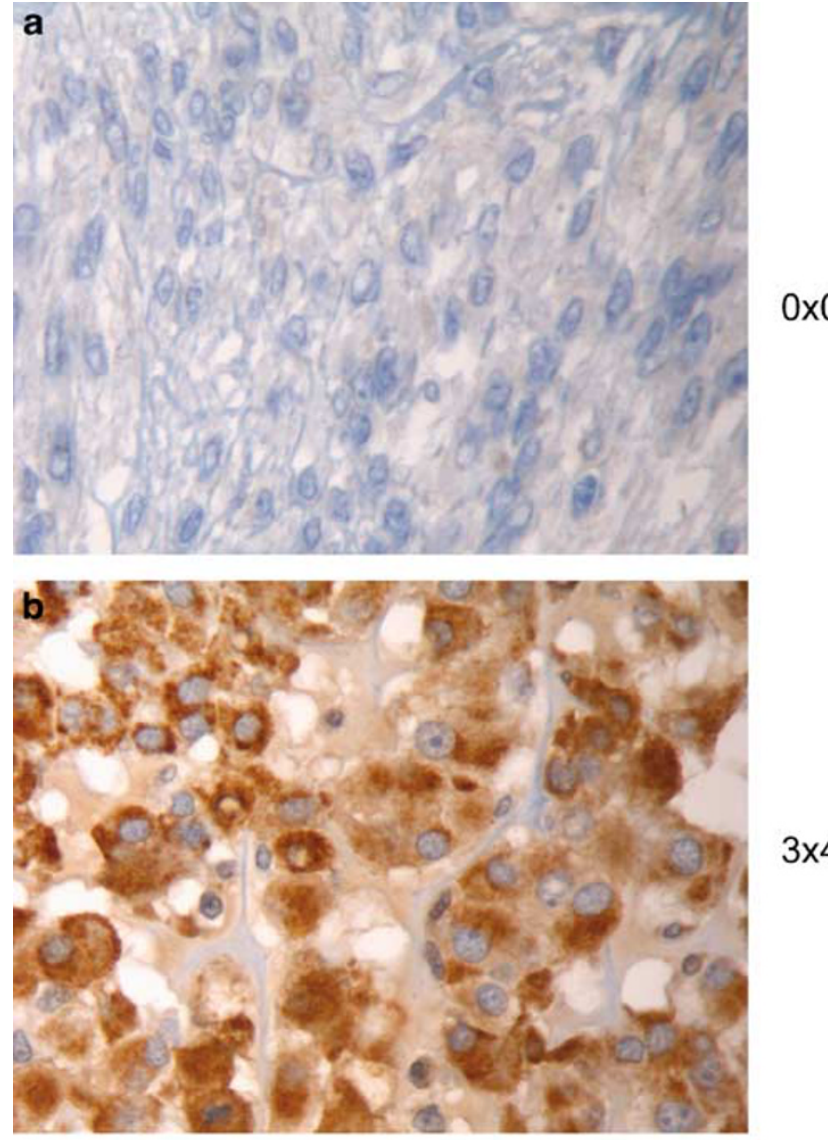

$3 \times 4$

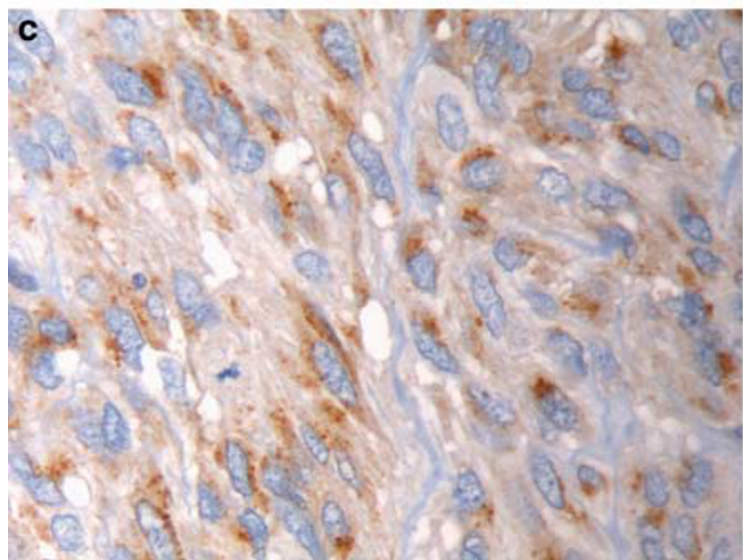

$2 \times 3$

Figure 1 Evaluation of staining intensity and number of stained cells. (a) GIST (with spindle cell features) with no staining in any of the cells, giving a score of 0 . (b) GIST (with epithelioid features) with strong staining intensity in $>80 \%$ of cells, giving a score of 12. (c) Leiomyosarcoma with moderate staining intensity in $50-80 \%$ of the cells, giving a score of 6 .

time of 3.4 years (Figure 2, $\log \operatorname{rank} P=0.015$ ). There was also a correlation between low IGF2-expressing tumors and gastric locations, which were more than twice as frequent compared with tumors in the small bowel $(P<0.001)$. Among the gastric tumors, the patients with IGF2 overexpression had a median survival time of 4.0 years compared with those with tumors lacking overexpression, who had a median survival time of 6.9 years $(P=0.039)$. 
In a multivariable analysis of all the 445 GIST patients, high mitotic count $(>5 / 50$ high power fields) and increased tumor size $(>5 \mathrm{~cm})$ were unfavorable prognostic factors $(P<0.001$ and $P=0.001)$. Localization of gastric tumor compared with all other sites was favorable in the same analysis $(P=0.018)$, but overexpression of IGF2 did not hold up as independently significant in the multivariate test $(P=0.188)$ against these established prognostic factors.

\section{Mesenchymal Tumors}

Results were obtained for 51 diagnostic classes of bone and soft-tissue tumors. The tumor type with the most prominent expression, in frequency and magnitude, was solitary fibrous tumor. Among those with $>10$ cases, synovial sarcomas, myxoid liposarcomas, GISTs, malignant peripheral nerve sheath tumors, chondrosarcomas, undifferentiated pleomorphic sarcomas (MFH), Ewing's sarcomas and tenosynovial giant cell tumors showed high level of expression in more than $20 \%$ of cases (Table 1a).

Chondrosarcomas were registered additionally by grade, with 30 cases in grade 1, 17 in grade 2 and 4 in grade 3 . The expression in grades 1 and 2 was the same with a median of 3.47 in both, but all 4 cases in the grade 3 category overexpressed IGF2 $(P=0.003)$. Synovial sarcomas fell into either grade 2 (62 cases) or grade 3 (18 cases), but there was no difference in the IGF2 expression between these groups (mean of 4.95 versus $4.46, P=0.7$ ).

Lower levels of expression $(10-20 \%$ of tumors meeting the criteria for overexpression) were present among dermatofibrosarcoma protuberans, extrauterine leiomyosarcomas and schwannomas. Little or no IGF2 overexpression was found in fibroblastic/ myofibroblastic and perivascular/vascular tumors. Among the classes with $\leq 10$ cases, pleomorphic liposarcomas and angiomyolipomas had high expression of IGF2 in more than half of the cases. Among rhabdomyosarcomas, four of seven embryonal (including infantile, pediatric and adult cases) and both pleomorphic rhabdomyosarcomas had high expression of IGF2, versus none of the four tested alveolar rhabdomyosarcomas. Other tumor types in which $>30 \%$ of cases had an expression score of 6 included well-differentiated liposarcomas, desmoplastic round cell tumors, clear cell sarcomas and chondroblastomas (Table 1b).

\section{Discussion}

The IGF1R is a growth factor receptor tyrosine kinase, with an extracellular ligand-binding domain, a transmembrane region and a cytoplasmic domain that contains a tyrosine kinase domain. ${ }^{36,37}$ Ligand binding is extracellular, but receptor activation is translated into the activation of intracellular domain functions. The sequences and structural characteristics of receptor tyrosine kinases is the basis for classifying them into subclasses. ${ }^{38,39}$ KIT belongs to group III together with platelet-derived growth factor receptor A and B, whereas the IGF1R belongs to group II subclass, together with the insulin receptor. The induced activation of the kinase domain is mediated by receptor oligomerization $^{40}$ and the phosphorylated receptors stimulate intracellular signaling pathways controlling cell proliferation, adhesion, apoptosis, survival and differentiation, primarily through the RAS-RAFMAPK and the PI3-K/AKT-mTOR cascades. ${ }^{17,41-43}$ The study of Hernando et $a l^{17}$ surveying the activation of these pathways in sarcomas found

Table 1a IGF2 expression in tumor groups represented by more than 10 cases

\begin{tabular}{|c|c|c|c|c|c|}
\hline Diagnosis & $\mathrm{n}$ & Positive & $\%$ & Mean score & $95 \% C I$ \\
\hline Solitary fibrous tumor & 25 & 20 & 80 & 7.4 & \pm 1.6 \\
\hline Synovial sarcoma & 80 & 34 & 43 & 4.9 & \pm 0.7 \\
\hline Myxoid liposarcoma & 33 & 13 & 39 & 4.6 & \pm 1.2 \\
\hline Gastrointestinal stromal tumor & 449 & 196 & 44 & 4.6 & \pm 0.3 \\
\hline Malignant peripheral nerve sheath tumor & 76 & 35 & 46 & 4.2 & \pm 0.8 \\
\hline Chondrosarcoma & 51 & 17 & 33 & 3.8 & \pm 0.9 \\
\hline Tenosynovial giant cell tumor & 34 & 8 & 24 & 3.7 & \pm 0.7 \\
\hline Undifferentiated pleomorphic sarcoma (MFH) & 65 & 21 & 32 & 3.6 & \pm 0.7 \\
\hline Ewing's sarcoma & 14 & 3 & 27 & 3.1 & \pm 1.5 \\
\hline Neurofibroma & 55 & 4 & 7 & 2.1 & \pm 0.5 \\
\hline Dermatofibrosarcoma protuberans & 17 & 2 & 12 & 1.7 & \pm 1.3 \\
\hline Leiomyosarcoma (extrauterine soft tissue) & 59 & 6 & 10 & 1.6 & \pm 0.6 \\
\hline Schwannoma & 42 & 5 & 12 & 1.4 & \pm 0.7 \\
\hline Endometrial stromal tumor & 12 & 0 & 0 & 1.3 & \pm 0.9 \\
\hline Osteosarcoma & 12 & 0 & 0 & 1.0 & \pm 0.7 \\
\hline Angiosarcoma & 13 & 0 & 0 & 0.9 & \pm 0.8 \\
\hline Fibroma (benign) & 28 & 0 & 0 & 0.8 & \pm 0.5 \\
\hline Hemangioendothelioma (epithelioid) & 12 & 0 & 0 & 0.5 & \pm 0.7 \\
\hline Fibromatosis (desmoid-type) & 23 & 0 & 0 & 0.4 & \pm 0.3 \\
\hline Fibroadenoma (breast) & 11 & 0 & 0 & 0.2 & \pm 0.4 \\
\hline
\end{tabular}


Table 1b IGF2 expression in tumor groups with 10 or fewer cases

\begin{tabular}{|c|c|c|}
\hline Diagnosis & $\mathrm{n}$ & $\begin{array}{c}\text { Cases overexpressing } \\
\text { IGF2 }\end{array}$ \\
\hline \multicolumn{3}{|l|}{ Adipocytic } \\
\hline Lipoma & 10 & 0 \\
\hline \multicolumn{3}{|l|}{ Liposarcoma } \\
\hline Well-differentiated & 10 & 4 \\
\hline Pleomorphic & 6 & 4 \\
\hline Angiomyolipoma & 8 & 5 \\
\hline \multicolumn{3}{|l|}{ Fibroblastic/myofibroblastic } \\
\hline $\begin{array}{l}\text { Fasciitis (nodular, cranial, } \\
\text { proliferative) }\end{array}$ & 9 & 0 \\
\hline Phylloides & 2 & 0 \\
\hline Digital fibromatosis & 4 & 0 \\
\hline Inflammatory pseudotumor & 3 & 0 \\
\hline Inflammatory myofibroblastic tumor & r 2 & 1 \\
\hline Myxoma & 7 & 2 \\
\hline Low-grade fibromyxoid sarcoma & 9 & 0 \\
\hline Fibrosarcoma & 2 & 0 \\
\hline \multicolumn{3}{|l|}{ Fibrohistocytic and histocytic tumor } \\
\hline Fibroxanthoma & 4 & 0 \\
\hline Atypical fibroxanthoma & 2 & 0 \\
\hline \multicolumn{3}{|l|}{ Muscle and related tumors } \\
\hline Leiomyosarcoma, uterine & 5 & 1 \\
\hline \multicolumn{3}{|l|}{ Rhabdomyosarcomas } \\
\hline Embryonal & 7 & 4 \\
\hline Alveolar & 4 & 0 \\
\hline Pleomorphic & 2 & 2 \\
\hline \multicolumn{3}{|l|}{ Perivascular/vascular tumors } \\
\hline Glomus tumor & 10 & 2 \\
\hline Hemangioma/lymphangioma & 7 & 0 \\
\hline Karposi sarcoma & 2 & 0 \\
\hline \multicolumn{3}{|l|}{ Peripheral nervous system tumors } \\
\hline Perineuroma & 4 & 1 \\
\hline \multicolumn{3}{|c|}{ Mesenchymal tumors of uncertain differentiation } \\
\hline $\begin{array}{l}\text { Desmoplastic small round cell } \\
\text { tumor }\end{array}$ & 6 & 2 \\
\hline Fibrous dysplasia & 10 & 1 \\
\hline Clear cell sarcoma & 7 & 3 \\
\hline $\begin{array}{l}\text { Extraskeletal myxoid } \\
\text { chondrosarcoma }\end{array}$ & 8 & 0 \\
\hline \multicolumn{3}{|l|}{ Bone tumors } \\
\hline Enchondroma & 5 & 0 \\
\hline Chondroblastoma & 4 & 2 \\
\hline Chondromyxoid fibroma & 2 & 1 \\
\hline \multicolumn{3}{|l|}{ Epithelial tumors } \\
\hline Carcinosarcoma & 7 & 0 \\
\hline Desmoplastic melanoma & 9 & 0 \\
\hline
\end{tabular}

evidence for massive upregulation not only of KIT, but also of IGF2 in GISTs. IGF2 was also overexpressed in synovial sarcoma, fibrosarcoma and myxoid/round cell liposarcoma. In our study, we had very few fibrosarcoma cases, but for synovial sarcomas, myxoid liposarcomas and GISTs, we found a relative overexpression of the IGF2 protein in $\sim 40 \%$ of the cases, among the highest levels seen in any of the $>50$ mesenchymal tumor types surveyed. In Hernando's study, there was no increased IGF2 gene expression in clear cell sarcomas, pleomorphic/de-differentiated liposarcomas, leiomyosarcomas or pleomorphic/undifferentiated sarcomas (MFH). For the two first categories, we had $<10$ cases and among the leiomyosarcomas, IGF2 protein overexpression was found in $\sim 10 \%$ of the cases. For the pleomorphic/undifferentiated sarcomas (MFH), we found protein overexpression in $>30 \%$ of the cases; the number of cases (10 cases in Hernando et al versus 65 in ours) might contribute to this discrepancy.

An earlier study correlating malignant potential with IGF expression in 46 soft-tissue sarcomas has been published, ${ }^{44}$ including 10 low-grade, 9 intermediate and 27 high-grade cases. A significant difference was found between the expression of IGF2 in the intermediate and the high-grade groups. In our material, there was also a significant difference in expression between chondrosarcoma grades 1 and 2 compared with that in grade 3 , but the number of cases in the latter was only four and can only be taken into account as a trend. The synovial sarcomas in our material showed no difference in the expression of IGF2 by grade, but were in general overexpressing IGF2 compared with many other tumor groups. It is noted that the tumor type with the highest expression (solitary fibrous tumor) is generally benign.

The ligands, IGF1 and IGF2, interact with IGF1R to promote growth. IGF2 can also bind to the IGF2R receptor, which has a growth inhibitory role by shunting IGF2 for lysosomal degradation, thereby decreasing circulating levels. Increased levels of circulating IGF2 (secretion from a tumor or reduced IGF2R receptor expression) can inhibit the secretion of growth hormone from the pituitary with secondarily decreased levels of IGF1 and insulin. ${ }^{13,21}$ The prohomone form of IGF2, 'big IGF2', released from mesenchymal tumors interacts poorly with IGF-binding proteins, thereby disproportionately increasing the level of unbound IGF, and has been reported to have a higher affinity for the insulin receptor than physiological IGF2. ${ }^{13}$ Severe hypoglycemia has been associated with non-islet-cell tumors and IGF2 is known to have such hypoglycemic effects presumably through insulin-like activity. The majority of the circulating IGF2 in non-islet-cell tumor hypoglycemia is big IGF2, and circulating high levels in a case with leiomyosarcoma and in cases with GISTs were reduced after surgery. ${ }^{13,21,23}$

The high expression of IGF2 protein in our study provides a biological explanation for case reports of GIST patients suffering from hypoglycemia. ${ }^{18-23}$ This phenomenon has been observed relatively frequently in association with solitary fibrous tumor, in which associated hypoglycemia has been termed 'Doege-Potter Syndrome',45,46 In GISTs, we also found that the expression of IGF2 correlated with increased size and higher mitotic count. This could 
Table 2 Clinicopathological characteristics of 445 GIST patients

\begin{tabular}{|c|c|c|c|}
\hline & All cases $(\mathrm{n}=445)$ & IGF2-positive cases $(\mathrm{n}=196)$ & IGF2-negative cases $(\mathrm{n}=249)$ \\
\hline \multicolumn{4}{|l|}{ Gender } \\
\hline Male & 231 & 107 & 124 \\
\hline Female & 214 & 89 & 125 \\
\hline Age at primary diagnosis, mean (range) & $64.9(23-94)$ & $64.3(25-93)$ & $65.3(23-94)$ \\
\hline \multicolumn{4}{|l|}{ Localization of primary tumor } \\
\hline Stomach & 229 & 83 & 146 \\
\hline Small bowel & 153 & 90 & 63 \\
\hline All other locations & 63 & 23 & 40 \\
\hline Size in $\mathrm{cm}$, mean (number of cases) & $8.2(333)$ & $8.4(151)$ & $8.1(182)$ \\
\hline Tumors $>5 \mathrm{~cm}$ & $195 / 333(59 \%)$ & $95 / 151(63 \%)$ & $100 / 182(55 \%)$ \\
\hline Mitoses/50 HPF, mean (range) & $9.1(0-276)$ & $9.2(0-92)$ & $8.9(0-276)$ \\
\hline Tumors with $>5$ mitosis/50 HPF & $143 / 445(32 \%)$ & $75 / 196(38 \%)$ & $68 / 249(27 \%)$ \\
\hline \multicolumn{4}{|l|}{ Risk of malignanc $\mathrm{y}^{\mathrm{a}}$} \\
\hline Very low potential & $24 \%$ & $17 \%$ & $28 \%$ \\
\hline Low potential & $15 \%$ & $14 \%$ & $18 \%$ \\
\hline Moderate potential & $26 \%$ & $26 \%$ & $22 \%$ \\
\hline High potential & $35 \%$ & $43 \%$ & $32 \%$ \\
\hline
\end{tabular}

${ }^{\mathrm{a}}$ Among 295 case with IGF2 staining data, complete size and mitotic count information.

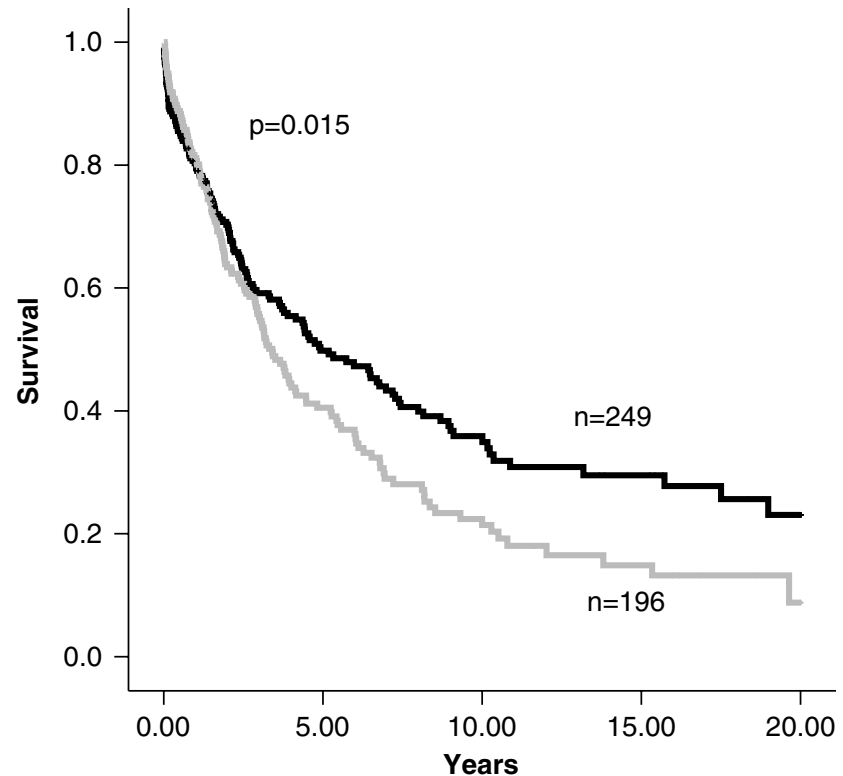

Figure 2 Overall survival for patients with GISTs expressing high IGF2 (bottom gray line, $n=196$ ) versus low IGF2 (top black line, $n=249$ ).

be because of simultaneous stimulation of multiple downstream pathways. Tarn et $a l^{47}$ have shown that the AKT pathway can be inhibited after treatment with tyrosine kinase inhibitors affecting the KIT receptor, keeping the MAPK pathway active. On the basis of this, an overexpression of IGF2 may cause a more aggressive cell phenotype resulting in larger tumors with a higher proliferation rate.
In synovial sarcoma, in vitro studies have found that the SS18-SSX oncogene induces IGF2 expression which seems critical to convey tumorigenic properties. ${ }^{48-50}$ IGF2 expression was associated with both the $-S S X 1$ and $-S S X 2$ translocation subtypes. ${ }^{48}$ In this study, we present evidence that in primary synovial sarcoma tumor specimens, IGF2 is highly expressed at the protein level, supporting translational relevance to patients of these experimental findings, and attempts to target this pathway in patients using IGF signaling inhibitors.

The association between IGFs and neoplasia has been a subject of investigation for many years. Under normal conditions, the balance between the expression and activities of these factors is tightly controlled, and changes in this balance may trigger events leading to malignancy. This knowledge has been the subject of intense interest because the IGF system can be a target for anti-cancer therapy. ${ }^{22}$ For example, Burtrum et $a 1^{51}$ have generated an antibody that binds IGF1R with high affinity and inhibits ligand binding, leading to the inhibition of downstream signaling in two of the major IGF pathways, and several humanized antibodies are now in clinical development. Tomizawa et $a 5^{52}$ were able to suppress the proliferation of hepatoblastoma cell lines by inhibiting the IGF1R with PPP, a commercially available tyrosine kinase inhibitor. Nielsen et $a I^{53}$ showed that urokinase plasminogen activator $(\mathrm{uPa})$ and IGF1R are highly expressed in breast cancer; inhibiting uPa resulted in the degradation of IGF1R and also inhibited the production of $u P A$ mRNA and protein in the presence of IGF1. mTOR, a 
major downstream effector of the IGF signaling pathway, is targeted by a new generation of rapamycin analogs, which are undergoing active evaluation in clinical trials. ${ }^{54}$ For GISTs in particular, a study on inhibiting IGF1R has shown promising results. ${ }^{55}$

In summary, following a broad examination of mesenchymal tumors by immunohistochemistry on tissue microarrays, expression of the growth factor and therapeutic target IGF2 is shown in solitary fibrous tumors, synovial sarcomas, myxoid liposarcomas, GISTs, malignant peripheral nerve sheath tumors, chondrosarcomas, undifferentiated pleomorphic sarcomas (MFH), Ewing's sarcomas and in tenosynovial giant cell tumors. High expression of IGF2 correlates with poor prognosis in GISTs, and is consistent with findings from gene expression studies in GIST and synovial sarcoma and also with reports of hypoglycemic complications in solitary fibrous tumors and GISTs.

\section{Acknowledgements}

TON is a Senior Scholar of the Michael Smith Foundation for Health Research. This research is supported by grants from the Canadian Institutes for Health Research and the Canadian Cancer Society. The tissue microarray facility at the Genetic Pathology Evaluation Centre is supported in part by an unrestricted educational grant from sanofi-aventis. We thank Erika Mehl for her technical assistance with immunohistochemistry and Samuel Leung for organizing the website. Work performed at Stanford University was supported by funding from the National Institute of Health (CA 112270).

\section{Conflict of interest}

The authors declare no conflict of interest.

\section{References}

1 D'Ercole AJ, Applewhite GT, Underwood LE. Evidence that somatomedin is synthesized by multiple tissues in the fetus. Dev Biol 1980;75:315-328.

2 Efstratiadis A. Genetics of mouse growth. Int J Dev Biol 1998;42:955-976

3 Khandwala HM, McCutcheon IE, Flyvbjerg A, et al. The effects of insulin-like growth factors on tumorigenesis and neoplastic growth. Endocr Rev 2000;21: $215-244$.

4 LeRoith D, Roberts Jr CT. The insulin-like growth factor system and cancer. Cancer Lett 2003;195:127-137.

5 Nijhout HF. The control of growth. Development 2003;130:5863-5867.

6 Smith FM, Garfield AS, Ward A. Regulation of growth and metabolism by imprinted genes. Cytogenet Genome Res 2006;113:279-291.
7 Jones JI, Clemmons DR. Insulin-like growth factors and their binding proteins: biological actions. Endocr Rev 1995;16:3-34.

8 Foulstone E, Prince S, Zaccheo O, et al. Insulin-like growth factor ligands, receptors, and binding proteins in cancer. J Pathol 2005;205:145-153.

9 Roche P, Brown J, Denley A, et al. Computational model for the IGF-II/IGF2r complex that is predictive of mutational and surface plasmon resonance data. Proteins 2006;64:758-768.

10 Cui H, Cruz-Correa M, Giardiello FM, et al. Loss of IGF2 imprinting: a potential marker of colorectal cancer risk. Science 2003;299:1753-1755.

11 Hankinson SE, Willett WC, Colditz GA, et al. Circulating concentrations of insulin-like growth factor-I and risk of breast cancer. Lancet 1998;351:1393-1396.

12 Wolk A, Mantzoros CS, Andersson SO, et al. Insulinlike growth factor 1 and prostate cancer risk: a population-based, case-control study. J Natl Cancer Inst 1998;90:911-915.

13 Daughaday WH, Emanuele MA, Brooks $\mathrm{MH}$, et al. Synthesis and secretion of insulin-like growth factor II by a leiomyosarcoma with associated hypoglycemia. N Engl J Med 1988;319:1434-1440.

14 Nielsen TO, West RB, Linn SC, et al. Molecular characterisation of soft tissue tumours: a gene expression study. Lancet 2002;359:1301-1307.

15 Segal NH, Pavlidis P, Antonescu CR, et al. Classification and subtype prediction of adult soft tissue sarcoma by functional genomics. Am J Pathol 2003;163:691-700.

16 Baird K, Davis S, Antonescu CR, et al. Gene expression profiling of human sarcomas: insights into sarcoma biology. Cancer Res 2005;65:9226-9235.

17 Hernando E, Charytonowicz E, Dudas ME, et al. The AKT-mTOR pathway plays a critical role in the development of leiomyosarcomas. Nat Med 2007;13: 748-753.

18 Pink D, Schoeler D, Lindner T, et al. Severe hypoglycemia caused by paraneoplastic production of IGF-II in patients with advanced gastrointestinal stromal tumors: a report of two cases. J Clin Oncol 2005;23:6809-6811.

19 Rikhof B, Van Den BG, van der Graaf WT. Non-islet cell tumour hypoglycaemia in a patient with a gastrointestinal stromal tumour. Acta Oncol 2005;44: 764-766.

20 Hamberg P, de Jong FA, Boonstra JG, et al. Non-isletcell tumor induced hypoglycemia in patients with advanced gastrointestinal stromal tumor possibly worsened by imatinib. J Clin Oncol 2006;24:e30-e31.

21 Fukuda I, Hizuka N, Ishikawa Y, et al. Clinical features of insulin-like growth factor-II producing non-islet-cell tumor hypoglycemia. Growth Horm IGF Res 2006;16: 211-216.

22 Guiteau J, Fanucchi M, Folpe A, et al. Hypoglycemia in the setting of advanced gastrointestinal stromal tumor. Am Surg 2006;72:1225-1230.

23 Escobar GA, Robinson WA, Nydam TL, et al. Severe paraneoplastic hypoglycemia in a patient with a gastrointestinal stromal tumor with an exon 9 mutation: a case report. BMC Cancer 2007;7:13.

24 Braconi C, Bracci R, Bearzi I, et al. Insulin-like growth factor (IGF) 1 and 2 help to predict disease outcome in GIST patients. Ann Oncol 2008;19:1293-1298.

25 Busund LT, Ow KT, Russell P, et al. Expression of insulin-like growth factor mitogenic signals in 
adult soft-tissue sarcomas: significant correlation with malignant potential. Virchows Arch 2004;444: 142-148.

26 Voduc D, Kenney C, Nielsen TO. Tissue microarrays in clinical oncology. Semin Radiat Oncol 2008;18: 89-97.

27 Yuen JS, Macaulay VM. Targeting the type 1 insulinlike growth factor receptor as a treatment for cancer. Expert Opin Ther Targets 2008;12:589-603.

28 Steigen SE, Straume B, Turbin D, et al. Clinicopathologic factors and nuclear morphometry as independent prognosticators in KIT-positive gastrointestinal stromal tumors. J Histochem Cytochem 2008;56:139-145.

29 Nielsen TO, Hsu FD, O’Connell JX, et al. Tissue microarray validation of epidermal growth factor receptor and SALL2 in synovial sarcoma with comparison to tumors of similar histology. Am J Pathol 2003;163:1449-1456.

$30 \mathrm{Ng}$ TL, Gown AM, Barry TS, et al. Nuclear beta-catenin in mesenchymal tumors. Mod Pathol 2005;18:68-74.

31 West RB, Harvell J, Linn SC, et al. Apo D in soft tissue tumors: a novel marker for dermatofibrosarcoma protuberans. Am J Surg Pathol 2004;28:1063-1069.

32 Terry J, Saito T, Subramanian S, et al. TLE1 as a diagnostic immunohistochemical marker for synovial sarcoma emerging from gene expression profiling studies. Am J Surg Pathol 2007;31:240-246.

33 Kobel M, Weichert W, Cruwell K, et al. Epithelial hyaluronic acid and CD44v6 are mutually involved in invasion of colorectal adenocarcinomas and linked to patient prognosis. Virchows Arch 2004;445:456-464.

34 Liu CL, Montgomery KD, Natkunam Y, et al. TMACombiner, a simple software tool to permit analysis of replicate cores on tissue microarrays. Mod Pathol 2005;18:1641-1648.

35 Hornick JL, Fletcher CD. The role of KIT in the management of patients with gastrointestinal stromal tumors. Hum Pathol 2007;38:679-687.

36 Hanks SK, Quinn AM, Hunter T. The protein kinase family: conserved features and deduced phylogeny of the catalytic domains. Science 1988;241:42-52.

37 Yarden Y, Ullrich A. Growth factor receptor tyrosine kinases. Annu Rev Biochem 1988;57:443-478.

38 Ullrich A, Schlessinger J. Signal transduction by receptors with tyrosine kinase activity. Cell 1990;61: 203-212.

39 Gschwind A, Fischer OM, Ullrich A. The discovery of receptor tyrosine kinases: targets for cancer therapy. Nat Rev Cancer 2004;4:361-370.

40 Williams LT. Signal transduction by the plateletderived growth factor receptor involves association of the receptor with cytoplasmic molecules. Clin Res 1989;37:564-568.

41 Lennartsson J, Blume-Jensen $\mathrm{P}$, Hermanson M, et al. Phosphorylation of Shc by Src family kinases is necessary for stem cell factor receptor/c-kit mediated activation of the Ras/MAP kinase pathway and c-fos induction. Oncogene 1999;18:5546-5553.

42 Duensing A, Medeiros F, McConarty B, et al. Mechanisms of oncogenic KIT signal transduction in primary gastrointestinal stromal tumors (GISTs). Oncogene 2004;23:3999-4006.

43 Goddard NC, McIntyre A, Summersgill B, et al. KIT and RAS signalling pathways in testicular germ cell tumours: new data and a review of the literature. Int J Androl 2007;30:337-348.

44 Busund LT, Ow KT, Russell P, et al. Expression of insulin-like growth factor mitogenic signals in adult soft-tissue sarcomas: significant correlation with malignant potential. Virchows Arch 2004;444:142-148.

45 Zafar H, Takimoto CH, Weiss G. Doege-Potter syndrome: hypoglycemia associated with malignant solitary fibrous tumor. Med Oncol 2003;20:403-408.

46 Balduyck B, Lauwers P, Govaert K, et al. Solitary fibrous tumor of the pleura with associated hypoglycemia: Doege-Potter syndrome: a case report. J Thorac Oncol 2006;1:588-590.

47 Tarn C, Skorobogatko YV, Taguchi T, et al. Therapeutic effect of imatinib in gastrointestinal stromal tumors: AKT signaling dependent and independent mechanisms. Cancer Res 2006;66:5477-5486.

48 Sun Y, Gao D, Liu Y, et al. IGF2 is critical for tumorigenesis by synovial sarcoma oncoprotein SYT-SSX1. Oncogene 2006;25:1042-1052.

49 de Bruijn DR, Allander SV, van Dijk AH, et al. The synovial-sarcoma-associated SS18-SSX2 fusion protein induces epigenetic gene (de)regulation. Cancer Res 2006;66:9474-9482.

50 Friedrichs N, Kuchler J, Endl E, et al. Insulin-like growth factor-1 receptor acts as a growth regulator in synovial sarcoma. J Pathol 2008;216:428-439.

51 Burtrum D, Zhu Z, Lu D, et al. A fully human monoclonal antibody to the insulin-like growth factor I receptor blocks ligand-dependent signaling and inhibits human tumor growth in vivo. Cancer Res 2003;63:8912-8921.

52 Tomizawa M, Saisho H. Signaling pathway of insulinlike growth factor-II as a target of molecular therapy for hepatoblastoma. World J Gastroenterol 2006;12: 6531-6535.

53 Nielsen TO, Andrews HN, Cheang M, et al. Expression of the insulin-like growth factor I receptor and urokinase plasminogen activator in breast cancer is associated with poor survival: potential for intervention with 17-allylamino geldanamycin. Cancer Res 2004;64:286-291.

54 Wan X, Helman LJ. The biology behind mTOR inhibition in sarcoma. Oncologist 2007;12:1007-1018.

55 Tarn C, Rink L, Merkel E, et al. Insulin-like growth factor 1 receptor is a potential therapeutic target for gastrointestinal stromal tumors. Proc Natl Acad Sci USA 2008;105:8387-8392. 